\title{
Comorbidity and diagnosing developmental disorders
}

Volume 4 Issue I - 2016

Robert Perna
Clinical neuropsychologist, TIRR Memorial Hermann, USA

Correspondence: Robert Perna, Ph.D. Clinical neuropsychologist, TIRR Memorial Hermann, Texas Medical Center Houston, 2450 Holcombe Blvd \#I, Houston, TX 7702I, USA,Tel 706-750-2572,Email dr.perna@juno.com

Received: January 0I, 1970 | Published: January 26, 2016 these include the fact that not only are many diagnoses not mutually exclusive, but having one may increase the risk of having particular other diagnoses. Moreover, diagnostic criteria and nomenclature have recently changed. For example, some diagnoses such as ADHD no longer have exclusionary criteria for people with Autism Spectrum disorder and some diagnoses such as Asperger's Disorder are no longer made. Many of the diagnoses listed in the DSM-5 and other sources tend to have diagnostic criteria based on constellations of behaviors or symptoms that may overlap with many different diagnoses.

Neurodevelopmental disorders are now known to have a higher incidence and higher rate of comorbidities than conditions more frequently than would be expected by chance. In the past, comorbidity was thought to be the exception whereas it now known to be the rule with several developmental disorders such as ADHD comorbid with anxiety disorder and other disorders. Understanding rates of comorbidity helps the differential diagnosis process. The diagnostic process involves determining if the symptoms are setting specific and if they are of sufficient severity to warrant a diagnosis. For some children the clinician must determine if the child has all the potential disorders that could be made based on their symptom presentation, or if there is a parsimonious explanation that could account for all the symptoms with the fewest possible diagnoses. Since many disorder have no real pathognomonic signs, it may be important for the clinician to keep track of how many assumptions they may be making and also to understand the comorbidities of each disorder and how having one disorder may increase the risk of other disorders.

The diagnostic process is even more difficult when considering how developmental disorders, early life brain injuries, and psychiatric disorders often have overlapping symptoms. The majority of neurodevelopmental disorders have a high incidence of comorbid conditions and there can be similar symptoms between different disorders. For example, some developmental disorder issues in the clinical literature suggest: ${ }^{1}$ that ADHD may look like Bipolar Disorder, Bipolar Disorder may be over diagnosed, genetic disorders are often misdiagnosed as Autism Spectrum Disorder, and that ADHD and Learning Disorders are often comorbid though clinicians may often assume that ADHD cause diminished academic performance when in fact it may be that the child has both disorders.

The assessing clinician must weigh the goal of diagnostic accuracy and parsimony against the high risk of comorbidity and the likelihood that the child with one developmental disorder may have a second or third. Also, the clinician must make a distinction between a behavioral and/or academic problem versus a diagnosable disorder may be very difficult. This issue may be even further complicated by clinicians feeling less bound by the DSM-V. Insight into the correct

diagnosis, helps drive appropriate and timely treatments and has great implications for these children. ${ }^{2}$ Especially since these disorders often overlap, differential diagnosis is necessary to provide appropriate services. In the past our diagnostic manuals (past versions of the DSM) precluded comorbid diagnoses of disorders such as autism and ADHD, this exclusionary criteria is no longer present in the proposed DSM-V. This is the recognition that although symptoms may overlap, a child with autism and ADHD is distinctly different from a child with autism alone; thus, may require different intervention services.

Research evidence suggests that $50-80 \%$ of children with any diagnosis meet criteria for at least one other diagnosis. ${ }^{3}$ The cooccurrence of ADHD and dyslexia is well established ${ }^{4}$ with the rates of overlap typically estimated from $30-50 \% .{ }^{5,6}$ Several studies have also demonstrated that children with ADHD display a high prevalence of language problems. ${ }^{7}$ Similarly, a number of studies have reported that many children with learning disabilities also display developmental coordination disorder. ${ }^{8}$

One study found ${ }^{9}$ found that $58 \%$ of our sample of children with ADHD also displayed reading disabilities and $27 \%$ of these children with ADHD had Developmental coordination disorder (DCD). Moreover, $82 \%$ of their sample with DCD had a comorbid disorder.

\section{Comorbidity with ADHD}

Attention Deficit Hyperactivity Disorder (ADHD) has a very high level of comorbidity and also is known to be difficult to differentially diagnosis from Bipolar disorder. The comorbidity of ADHD with other disorders is between $60 \%$ and $80 \%$ Some research has suggested that the attention issues contribute to the development of reading skills, however, several cognitive deficits, including processing and naming speed, were later shown to be shared between the disorders, ${ }^{10}$ in line with a common etiology model. A comorbid diagnosis of ADHD and depression occurs in approximately $20 \%$ to $30 \%$ of individuals, and ADHD and anxiety in more than $25 \%$ of people. ${ }^{11}$ Some research suggests that approximately $20 \%$ of adults with Bipolar disorder may also ADHD. ${ }^{12}$ 


\section{Comorbidities with learning disorders}

Learning disorders are also known to have a high rate of comorbidy with an estimated $60 \%$ of individuals with reading disorder also meeting the diagnostic criteria for at least one neuropsychiatric disorder. ${ }^{13}$ Estimates of the comorbidity between reading disorder and ADHD have varied greatly between samples and ranged from $10 \%$ to $50 \% .{ }^{14}$ Margari and colleagues ${ }^{15}$ analyzed sample of individuals with learning disorders and they found that Language Disorder was present in $28.6 \%$, and Developmental Coordination Disorder in 27.5\%.

\section{Conclusion}

The appropriate diagnosis and treatment of childhood disorders can change the entire trajectory of a childs' education and life. Some of the most common developmental disorder such as ADHD and Learning disorders have very high rates of comorbidy. Misdiagnosis is avoidable and its cost incalculable. Understanding disorder base rates, comorbidity rates and understanding the need for great diligence in adhering to strict diagnostic criteria is essential.

\section{Acknowledgments}

None.

\section{Conflicts of interest}

The authors declare there is no conflict of interests.

\section{Funding}

None.

\section{References}

1. Kaplan A. Anxiety disorder and ADHD: comorbitidy the rule not the exception, Psychiatric Times. 2012.

2. McDonald-McCary L, Grefer M, Mounts M, et al. The importance of differential diagnosis in neurodevelopmental disorders:Implications for IDEA, The School Psychologist. 2012.

3. Biederman J, Faraone SV, Keenan K, et al. Family-genetic and psychosocial risk factors in DSM-III attention deficit disorder. $J$ Am Acad Child Adolesc Psychiatry. 1990;29(4):526-533.
4. Gilger JW, Pennington BF, DeFries JC. A twin study of the etiology of comorbidity: attention-deficit hyperactivity disorder and dyslexia. $J \mathrm{Am}$ Acad Child Adolesc Psychiatry. 1992;31(2):343-348.

5. Dykman RA, Ackerman PT. Attention deficit disorder and specific reading disability:Separate but often overlapping disorders. J Learn Disabil. 1991;24(2):96-103.

6. Semrud-Clikeman M, Biederman J, Sprich-Buckminster S, et al. Comorbidity between ADHD and learning disability:A review and report in a clinically referred sample. $J$ Am Acad Child Adolesc Psychiatry. 1992;31(3):439-448.

7. Carte ET, Nigg JT, Hinshaw SP. Neuropsychological functioning motor speed and language processing in boys with and without ADHD. $J$ Abnorm Child Psychol. 1996;24(4):481-498.

8. Sugden DA, Wann C. The assessment of motor impairment in children with moderate learning difficulties. British Journal of Educational Psychology. 1987;57(2):225-236.

9. Dewey D, Wilson B, Crawford SG, et al. Comorbidity of developmental coordination disorder with ADHD and reading disability. Journal of the International Neuropsychological Society. 2000;6:152.

10. Willcutt EG, Pennington BF, Boada R, et al. A comparison of the cognitive deficits in reading disability and attention-deficit/hyperactivity disorder. J Abnorm Psychol. 2001;110(1):157-172.

11. Michielsen M, Comijs HC, Semeijn EJ, et al. The comorbidity of anxiety and depressive symptoms in older adults with attentiondeficit/hyperactivity disorder:A longitudinal study. J Affect Disord. 2013;148(2-3):220-227.

12. Perugi G, Ceraudo G, Vannucchi G, et al. Attention deficit/hyperactivity disorder symptoms in Italian bipolar adult patients:a preliminary report. $J$ Affect Disord. 2013;149(1-3):430-434.

13. Trzesniewski KH, Moffitt TE, Caspi A, et al. Revisiting the association between reading achievement and antisocial behavior:new evidence of an environmental explanation from a twin study. Child Dev. 2006;77(1):72-88.

14. Langberg JM, Vaughn AJ, Brinkman WB, et al. Clinical utility of the Vanderbilt ADHD Rating Scale for ruling out comorbid learning disorders. Pediatrics. 2010;126(5):1033-1038.

15. Margari L, Buttiglione M, Craig F, et al. Neuropsychopathological comorbidities in learning disorders. BMC Neurol. 2013;13:198. 